\title{
Utilisation de Rulactine en tant qu'agent d'affinage dans des fromages à pâte pressée
}

\author{
W. ALKHALAF *, L. VASSAL *, M.J. DESMAZEAUD ** \\ et J.-C. GRIPON * \\ avec la collaboration de Elisabeth PERROT *, G. PITEL *, J. URO * \\ et R.LE GOUAR * \\ * I.N.R.A., Laboratoire de Biochimie et Technologie laitières, 78350 Jouy-en-Josas, France \\ ** I.N.R.A., Laboratoire de Microbiologie laitière, 78350 Jouy-en-Josas, France
}

\section{Résumé}

A la dose de 450 Unités/1 l'addition de Rulactine (métalloprotéase synthétisée par Micrococcus caseolyticus) au lait servant à la fabrication de fromages à pâte pressée entraîne une intensification nette de la protéolyse et une modification de la texture de la pâte qui devient plus friable. On aboutit cependant à l'apparition d'amertume après $30 \mathrm{j}$ d'affinage et à des pertes de rendement non négligeables au cours de la fabrication du caillé. Les diagrammes d'électrophorèse des caséines des fromages contenant une dose de 450 Unités montrent une dégradation plus intense de la caséine $\beta$ et l'apparition nette d'une bande de faible mobilité électrophorétique. Cette bande caractéristique résulte de l'hydrolyse de la caséine $\beta$. A la dose de 90 Unités/1 de lait aucune différence n'a été observée entre les fromages essais et les témoins.

Mots clés: Accélération de l'affinage - Fromages à pâte pressée - Protéolyse Amertume - Texture - Protéase - Rulactine.

\section{Summary}

Use of Rulactine as ripening agent in semi-hard cheese

Addition of 450 Units/1 of Rulactine (metalloprotease from Micrococcus caseolyticus) in milk used for semi-hard cheesemaking developed an intensification of the proteolysis and a modification of texture of the curd. Thus, curds with Rulactine were more crumbly than controls. With 450 Units/1 of milk bitterness is occurring after 30 days of ripening and cheese yields were decreasing during cheesemaking. Electrophoregrams of curd-casein showed (for 450 Units dose) a significant increase of $\beta$-casein breakdown and the presence of a new band with a low electrophoretic mobility. This specific band results of $\beta$-casein hydrolysis. At the level of 90 Units/1 of milk no difference have been observed between the assay and the control cheeses.

Key words: Acceleration of ripening - Semi-hard cheese proteolysis - Bitterness Texture - Protease - Rulactine. 


\section{Introduction}

L'affinage est un processus complexe au cours duquel de nombreuses modifications biochimiques interviennent. Dans le cas des fromages à pâte ferme, la durée de l'affinage entraîne des immobilisations de capitaux qui se répercutent sur les coûts de production. Plusieurs méthodes ont été proposées pour améliorer l'affinage notamment l'élévation de la température des caves, l'addition de levains modifiés par traitement thermique ou chimique ou l'addition de préparations enzymatiques (LAw, 1984).

L'addition de protéase a été testée dans le cas du Cheddar afin d'accélérer la formation du goût (Sood et KosIKowsKI, 1979). Des résultats positifs ont été obtenus par l'addition d'une métalloprotéase (Neutrase) utilisée soit seule (LAW et WIGMORE, 1982) soit en association avec des extraits de bactéries lactiques (LAW et Wigmore, 1983).

En France, l'emploi d'une métalloprotéase synthétisée par Micrococcus caseolyticus et aux propriétés voisines de la Neutrase (Desmazeaud et HerMIER, 1968a et b) a été suggéré. Les premiers essais avec cette protéase en industrie fromagère ont été effectués en fabrication de fromage à pâte cuite pressée (GRUDE, 1978). L'enzyme était ajoutée directement au lait et les peptides produits durant sa maturation exerçaient un effet stimulant sur les streptocoques thermophiles. La régularité de l'acidification était améliorée et une plus faible proportion de fromages de qualité inférieure était observée. L'enzyme résiduelle dans le caillé était également supposée accélérer la dégradation des protéines durant l'affinage et contribuer à une amélioration de la qualité (Grude, 1978).

L'utilisation de cette enzyme a été ensuite préconisée dans les fabrications de pâte pressée non pour produire des peptides stimulants mais en tant qu'agent d'affinage. Dans ce cas, l'enzyme est ajoutée directement au lait de fabrication à une dose de 90 Unités (mesurées selon Desmazeaud et Hermier, 1968a) par litre de lait sans aucune modification du procédé de fabrication. Dans certaines usines, l'apparition d'une texture typique était observée plus rapidement sans qu'il y ait modification du goût et de l'arôme des produits. Toutefois, dans d'autres usines, aucun effet n'a pu être détecté.

Nos connaissances sur l'action de la Rulactine au cours de la fabrication du fromage sont très limitées (VASSAL et al., 1982) ; aussi les travaux présentés ici, ont été entrepris dans le but de mesurer son taux de rétention dans le caillé et de définir l'influence de cette enzyme sur le niveau de protéolyse, sur les pertes en protéines dans le sérum, sur les modifications de la texture et l'apparition éventuelle d'amertume. 


\section{Matériel et méthodes}

\section{A. Fabrication des fromages}

Des fromages à pâte pressée de type Saint-Paulin et de petit format $(250 \mathrm{~g})$ ont été fabriqués. Après pasteurisation $\left(72^{\circ} \mathrm{C}, 15 \mathrm{~s}\right)$, le lait était ensemencé à $2 \%$ avec un levain de Streptococcus cremoris CNRZ 112. Le caillé était partiellement délactosé par addition d'eau après soutirage de sérum $(30 \%)$. Après salage ces fromages étaient enrobés de cire. Leur extrait sec était proche de $53 \%$ et leur rapport gras/sec de $45 \%$. Pour les fromagesessais, la Rulactine était ajoutée directement au lait juste avant l'emprésurage. Les doses de Rulactine utilisées étaient de 45, 90, 225, 450, 900, 1800 et 2700 Unités/litre de lait (respectivement doses 0,$5 ; 1 ; 2,5 ; 5 ; 10 ; 20$ et 30 ). Ces fromages étaient affinés à $12{ }^{\circ} \mathrm{C}$ et $95 \%$ d'humidité relative jusqu'à 60 jours. La Rulactine nous a été gracieusement fournie par la Société Roussel-Uclaf (France).

\section{B. Dispositif pour mesurer les pertes d'azote dans le lactosérum}

Deux kilos de lait pasteurisé $\left(72^{\circ} \mathrm{C}, 15 \mathrm{~s}\right)$ étaient placés dans une cuve munie d'un couvercle et thermostatée à $32^{\circ} \mathrm{C}$. Le lait était inoculé avec $1 \%$ d'une culture de $S$. cremoris CNRZ 112 additionné d'une dose de Rulactine préalablement dissoute dans $10 \mathrm{ml}$ d'eau distillée, puis de $4 \mathrm{ml}$ de présure commerciale Boll, diluée 10 fois. Le caillé était découpé en cubes de $1 \mathrm{~cm}$ de côté, $30 \mathrm{mn}$ après l'addition de présure. Trente minutes après ce découpage, on prélevait $500 \mathrm{ml}$ de lactosérum, puis on plaçait le caillé sur un filtre et le lactosérum était collecté toutes les $30 \mathrm{mn}$ pendant $3 \mathrm{~h}$, puis toutes les heures pendant 3 nouvelles heures. Un dernier prélèvement avait lieu à $24 \mathrm{~h}$. Cet égouttage était réalisé à $32{ }^{\circ} \mathrm{C}$, dans des conditions qui évitaient l'évaporation.

\section{Détermination du taux de rétention de la Rulactine dans les caillés}

Le taux de rétention a été mesuré à l'aide d'une préparation de Rulactine marquée au ${ }^{14} \mathrm{C}$. Le marquage était effectué avec du formaldéhyde ${ }^{14} \mathrm{C}$ selon une méthode décrite par DoNNElly et al. (1980). La radioactivité spécifique de la Rulactine marquée était de $84 \cdot 10^{-3} \mu \mathrm{Ci} / \mathrm{mg}$. 4,2 mg de Rulactine marquée, préalablement mis en solution dans $1,4 \mathrm{ml}$ de Tris-HC1 $50 \mathrm{mM}, \mathrm{pH} 7,0$, étaient dilués dans $400 \mathrm{ml}$ de lait utilisés pour la fabrication de fromagesmodèles selon une technique décrite précédemment (PIARD et al., 1986). Après pressage, $10 \mathrm{~g}$ de caillé étaient mis en suspension à l'aide d'un Ultraturrax à $50{ }^{\circ} \mathrm{C}$ dans $40 \mathrm{ml}$ de solution de citrate de sodium, $0,5 \mathrm{M}, \mathrm{pH} 7,0$. Le volume final de la solution était ajusté à $200 \mathrm{ml}$. La radioactivité était ensuite dosée sur $1 \mathrm{ml}$ de la suspension de caillé ou sur $1 \mathrm{ml}$ de lactosérum par scintillation liquide à l'aide d'un compteur BECKMAN LS 2800.

\section{Analyses}

Le $\mathrm{pH}$ des fromages était mesuré directement dans le fromage à l'aide d'une électrode combinée à aiguille. 
L'azote total était mesuré à partir d'une solution citratée à $10 \mathrm{~g}$ de fromage pour $200 \mathrm{ml}$. Les fractions azotées solubles à pH 4,6 (NS), solubles dans l'acide trichloracétique à $12 \%$ (NPN) et solubles dans l'acide phosphotungstique à $2,5 \%$ (NAPT), étaient préparées à partir de cette solution citratée selon les techniques décrites précédemment (Gripon et al., 1975). La teneur en azote était déterminée après minéralisation par dosage colorimétrique de l'ammoniac selon Koops et al. (1975). Pour les essais concernant les cinétiques des pertes d'azote au cours de l'égouttage, les dosages ont été effectués à l'aide d'un autoanalyseur Technicon II.

L'étude qualitative de la protéolyse des caséines a été conduite par électrophorèse en gel d'acrylamide-agarose selon une technique décrite précédemment (Gripon et al., 1975).

\section{E. Mesures rhéologiques}

La texture était appréciée à l'aide d'un appareil Instron dans lequel une éprouvette de fromage était comprimée entre une plaque fixe et une plaque mobile qui descendait à une vitesse constante $(25 \mathrm{~mm} / \mathrm{mn})$. Les échantillons étaient de forme cylindrique ( $24 \mathrm{~mm}$ de diamètre) et d'une hauteur de $24 \mathrm{~mm}$. La compression était poursuivie jusqu'à ce que la hauteur de l'échantillon soit égale à $20 \%$ de sa hauteur initiale. La force subie par l'échantillon était enregistrée en continu. Ces opérations étaient effectuées dans un local maintenu à $21^{\circ} \mathrm{C}$, sur des échantillons stabilisés à cette température.

\section{F. Analyse sensorielle}

L'amertume des fromages était déterminée par un jury d'environ 10 dégustateurs bien entraînés qui notaient l'intensité du défaut sur une échelle de 0 à 10. Pour chaque dose de Rulactine, les dégustations étaient organisées suivant un plan en blocs incomplets équilibrés, qui comportait trois répétitions par traitement. Les dégustations avaient lieu après 15,30 et 45 jours d'affinage.

\section{Résultats}

\section{A. Influence sur le $p H$ du lactosérum}

La différence de $\mathrm{pH}$ du lactosérum entre les essais et les témoins (dispositif décrit par. II.B) était négligeable au début de l'égouttage, puis elle augmentait à la fin de celui-ci et restait constante jusqu'à $24 \mathrm{~h}$. La diminution de $\mathrm{pH}$ était de 0,25 unité dans le cas d'une dose 10 , de 0,13 unité dans le cas de la dose 5. Dans l'essai avec la dose 1, cette différence était toujours inférieure à 0,05 unité $\mathrm{pH}$.

\section{B. Rétention de la Rulactine dans les caillés}

Le pourcentage de rétention de la Rulactine dans les caillés et son écarttype, étaient de $13,3 \pm 0,55$ ( 3 répétitions) et le pourcentage observé dans le lactosérum était de $87,3 \pm 0,72$ (3 répétitions). 


\section{Protéolyse au cours de l'égouttage. Conséquences sur le rendement}

Les concentrations en azote total dans le lactosérum filtré et centrifugé sont plus élevées dans les essais avec Rulactine, les différences étant plus marquées avec les doses 5 et 10 (fig. 1). Du fait du ralentissement de l'égouttage au cours du temps, les quantités cumulées d'azote total dans le lactosérum n'augmentent que lentement entre 6 et $24 \mathrm{~h}$. Les différences entre témoin et essai s'établissent essentiellement au cours des 6 premières heures d'égouttage proportionnellement à la dose de Rulactine utilisée.
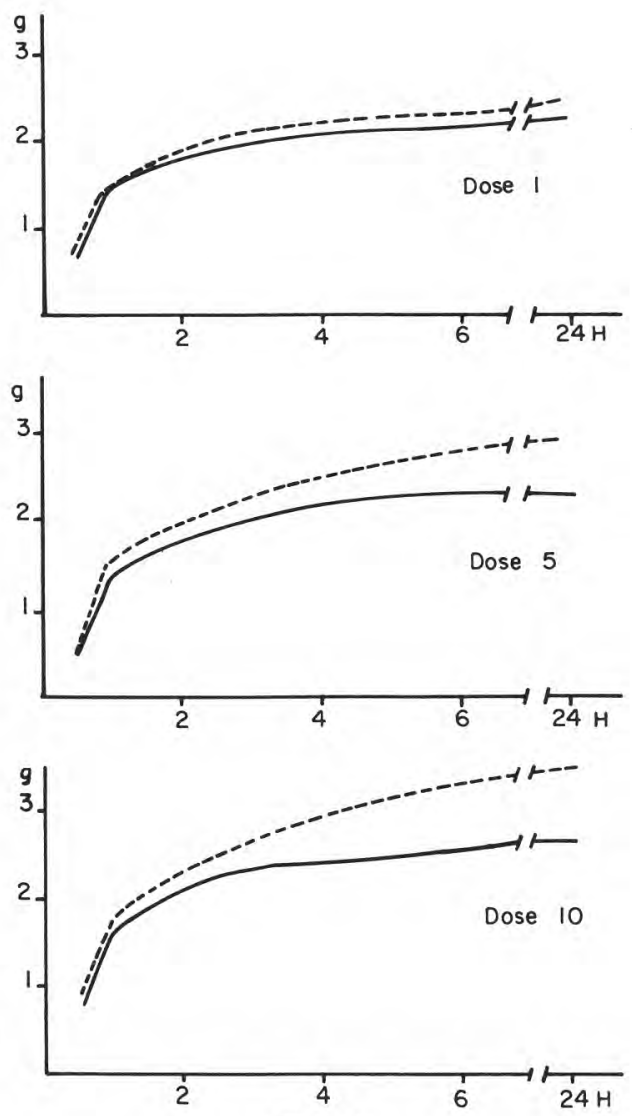

Fig. 1

Evolution de la concentration en azote dans le lactosérum au cours de la fabrication (les résultats sont exprimés en quantité cumulée obtenue à partir de deux litres de lait).

Evolution of the nitrogen amount in whey during cheesemaking (results are expressed as cumulative quantity from two liters of milk).

- - Fabrications avec différentes doses de Rulactine

Curds with various amount of Rulactine

Fabrications témoins

Control curds 
Les pourcentages d'azote retenu dans le caillé sont donnés dans le tableau 1. Les différences entre essais et témoin sont très nettes dans le cas des doses 5 et 10. Par contre, dans le cas de la dose 1, la différence reste inférieure à $0,6 \%$.

Le rendement fromager corrigé pour les variations de teneur en extrait sec (Maubois et Mocquot, 1969) a été déterminé pour les doses 1 et 5 au cours de deux fabrications. La différence avec les témoins n'était pas interprétable avec la dose 1, par contre une diminution de rendement de l'ordre de 3,5\% était observée avec la dose 5 .

TABLEAU I

Pourcentage de matière azotée du lait restant dans le fromage Percentage of milk nitrogen measured in the curds

\begin{tabular}{c|c|c|c}
\hline Dose de Rulactine & Témoin & Rulactine & Différence \\
\hline \multirow{2}{*}{$900 \mathrm{U} / 1$ (dose 10) } & 75,33 & 67,86 & 7,47 \\
& 73,86 & 67,13 & 6,73 \\
& 74,13 & 65,14 & 8,99 \\
\hline \multirow{2}{*}{$450 \mathrm{U} / 1$ (dose 5) } & 74,26 & 72,65 & 1,61 \\
& 74,63 & 71,93 & 2,70 \\
& 71,98 & 69,31 & $+0,27$ \\
\hline \multirow{2}{*}{ U0 U/1 (dose 1) } & 73,33 & 73,06 & $+0,59$ \\
& 73,04 & 72,45 & $-0,04$ \\
& 71,68 & 71,72 & $-0,44$ \\
& 73,19 & 73,63 & $+0,38$ \\
\hline
\end{tabular}

\section{Protéolyse au cours de l'affinage}

Pour les différentes fractions azotées étudiées, aucune différence n'a pu être observée entre le témoin et la dose 1. Par contre, avec la dose 5 , on observe une protéolyse plus élevée. Cette différence de protéolyse est détectable dès $48 \mathrm{~h}$ et représente $4,3 \%$ de l'azote total (fig. 2). Elle s'amplifie au cours de l'affinage pour représenter $7 \%$ après $30 \mathrm{j}$. Il faut noter que la forte différence de protéolyse détectable dès $48 \mathrm{~h}$, traduit une forte activité de la Rulactine au cours de la préparation du caillé, les températures et le $\mathrm{pH}$ étant alors plus favorables à son action qu'au cours de l'affinage. Avec la dose 2,5, la protéolyse est plus développée que dans le témoin. Toutefois, la différence à $48 \mathrm{~h}$ est très faible mais s'amplifie lentement au cours de l'affinage pour atteindre respectivement 1,5 et $2 \%$ après $30 \mathrm{j}$ et $60 \mathrm{j}$.

L'évolution des valeurs du NPN est comparable à celle de l'azote soluble à $\mathrm{pH} 4,6$. Par contre, l'évolution des valeurs de l'azote soluble dans l'acide phosphotungstique est la même dans tous les fromages et indique que la Rulactine ne produit pas ou très peu de petits peptides et d'acides aminés libres (fig. 2). 


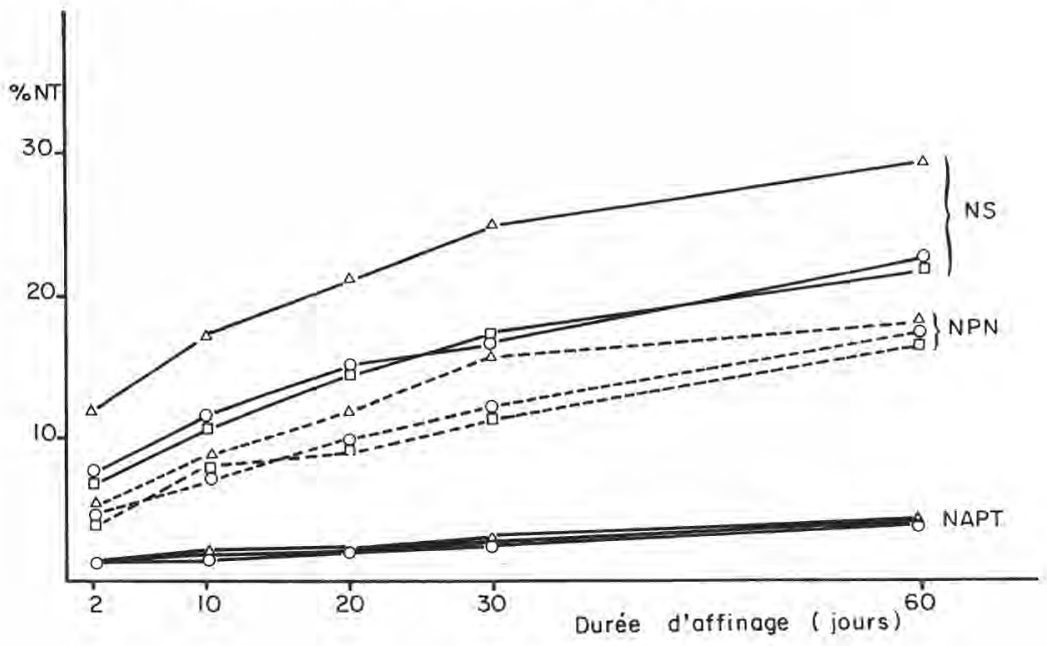

Fig. 2

Evolution des différentes fractions azotées au cours de l'affinage des fromages ayant reçu une dose $5(\Delta-\Delta)$ ou une dose $1(\square-\square)$ de Rulactine. $\mathrm{O}-\mathrm{O}=$ fromage-témoin.

$N S=$ azote soluble à $p H 4,6 ; N P N=$ azote non protéique soluble dans l'acide trichloracétique à $12 \% ; N A P T=$ azote soluble dans l'acide phosphotungstique.

Evolution of various nitrogen fractions during cheese ripening from curds after addition of dose $5(\Delta-\Delta)$ or dose $1(\square-\square)$ of Rulactine. $\mathrm{O}-\mathrm{O}=$ control curd.

$N S=p H 4.6$ soluble nitrogen $; N P N=$ non protein nitrogen soluble in $12 \%$ trichloracetic acid ; NAPT $=$ soluble nitrogen in phosphotungstique acid.

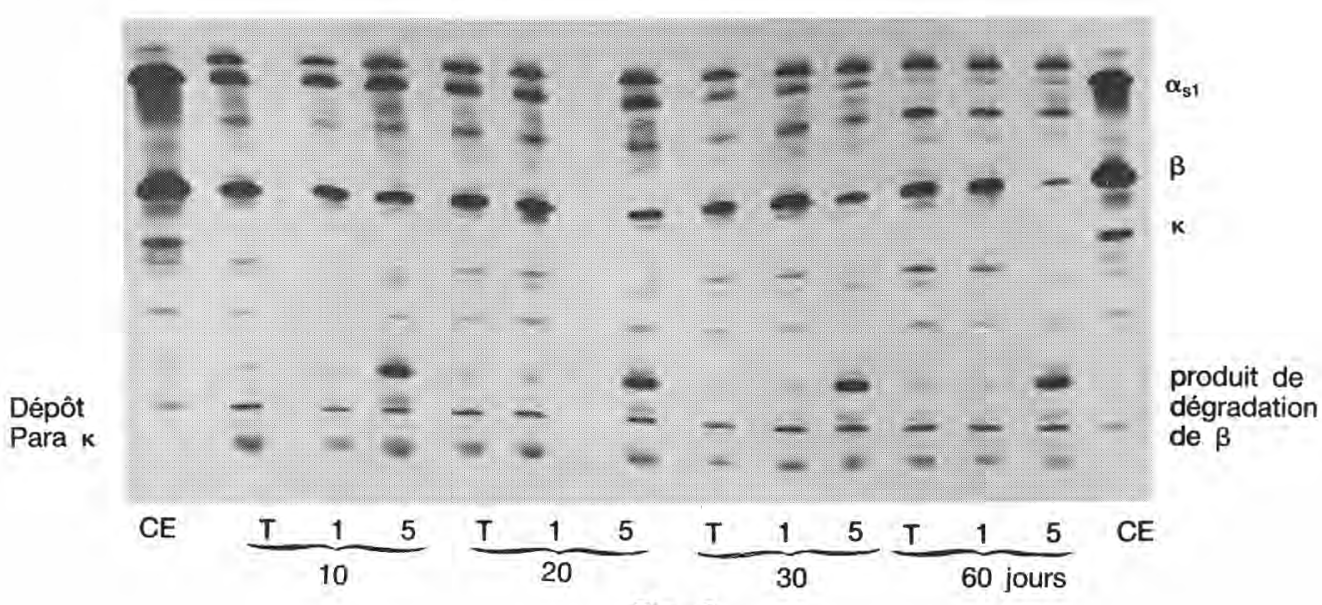

Fig. 3

Evolution des diagrammes électrophorétiques au cours de l'affinage des fromages ayant reçu une dose 5 (450 Unités/litre de lait) ou une dose 1 (90 U/l) de Rulactine et des fromages-témoins.

Evolution of electrophoregrams during cheese ripening of control and Rulactine curd (doses 5 and 1). 
Les diagrammes d'électrophorèse (fig. 3) ne révèlent pas de différence entre les fromages témoins et les fromages essais avec la dose 1, ceci même après $60 \mathrm{j}$ d'affinage. Avec les fromages contenant la dose 5 on observe une dégradation plus intense de la caséine $\beta$ et l'apparition nette d'une bande de faible mobilité électrophorétique (inférieure à celle des caséines $\gamma$ ). Ces modifications s'amplifient au cours de l'affinage. Elles étaient détectables dès $48 \mathrm{~h}$. Des électrophorégrammes analogues étaient observés avec la dose 2,5 mais les variations par rapport au témoin étaient moins marquées qu'avec la dose 5 (résultats non présentés). Au niveau des caséines $\alpha_{s 1}$ et $\alpha_{s 1} \mathrm{I}$, on n'observe aucune différence avec les témoins, même en présence de la dose 5.

L'hydrolyse in vitro de la caséine $\beta$ purifiée, par la Rulactine, révèle l'apparition d'une bande majeure de même mobilité électrophorétique que celle observée dans les fromages avec la dose 5 . Ceci suggère que le principal produit mis en évidence dans les fromages résulte de l'hydrolyse de la caséine $\beta$ (fig. 4).

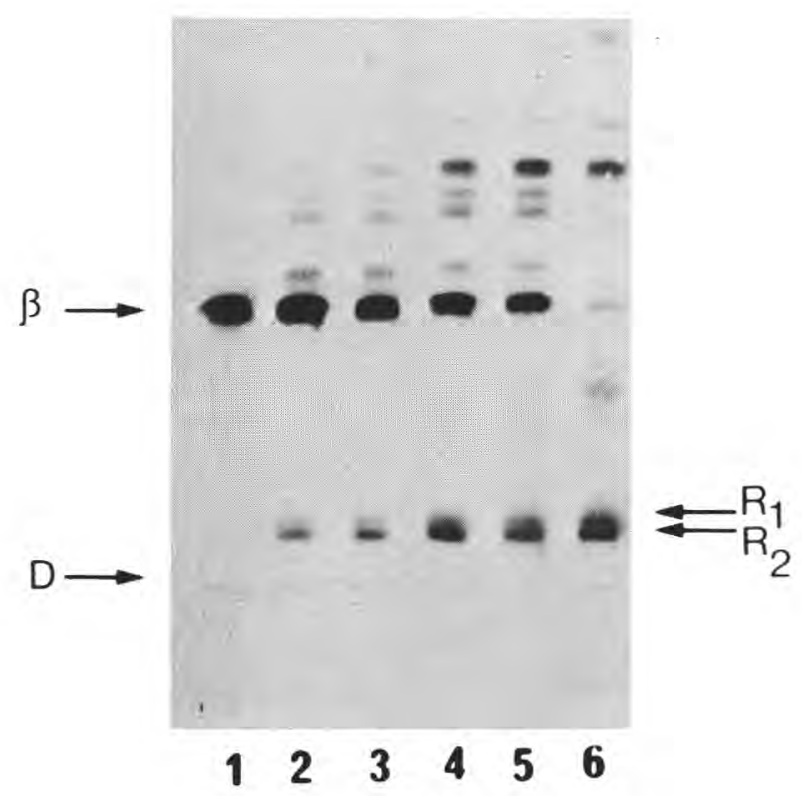

Fig. 4

Diagramme électrophorétique d'hydrolysats in vitro de caséine $\beta$ par la Rulactine.

$\mathrm{La}$ caséine $\beta$ était en solution à $2 \%$ dans un tampon Tris- $\mathrm{HCl}, \mathrm{pH}$ 7,5. Le rapport Rulactine-substrat était de $1 / 100$.

$1=$ Caséine $\beta$. $2 ; 3 ; 4 ; 5 ; 6=$ Caséine $\beta+$ Rulactine après $2 ; 5 ; 10 ; 20 ; 30 \mathrm{mn}$ d'hydrolyse à $37^{\circ} \mathrm{C} . R_{1}$ et $R_{2}=$ Produits d'hydrolyse majeurs.

Electrophoregram of $\beta$-casein in vitro hydrolysates by Rulactine.

$\beta$-casein was solubilized at $2 \%$ in Tris- $\mathrm{HCl}, \mathrm{pH} 7.5$ buffer. The Rulactin-substrate ratio was $1 / 100$.

1 : $\beta$-casein. $2 ; 3 ; 4 ; 5 ; 6=2 ; 5 ; 10 ; 20 ; 30 \mathrm{mn} \beta$-casein hydrolysis by Rulactine at $37^{\circ} \mathrm{C} . R_{1}$ and $R_{2}=$ specific hydrolysis products. 


\section{E. Propriétés rhéologiques des fromages}

Les courbes-types observées lors des tests de compression sont représentées figure 5 . On observe tout d'abord une augmentation régulière de la force nécessaire pour comprimer l'échantillon à vitesse constante, puis une diminution brutale de cette force correspondant à la rupture de l'échantillon. Cette diminution est suivie d'une nouvelle augmentation de cette force pour les taux de compression élevés. Dans les fromages en présence d'une dose 5 de Rulactine, la rupture se produit avec une force moins élevée et un taux de compression plus faible que dans les fromages témoins, ce qui traduit une plus grande friabilité de la pâte. Avec les doses 1 ou 2,5, les courbes de compression ne sont pas significativement différentes de celles des témoins. Les figures $6 \mathrm{~A}$ et $6 \mathrm{~B}$ présentent l'évolution de la force de rupture et du pourcentage de déformation à la rupture au cours de l'affinage. Les différences entre essais et témoins sont présentes dès $2 \mathrm{j}$ et révèlent une plus grande friabilité de la pâte des fromages-essais, qui persiste jusqu'à la fin de l'affinage.

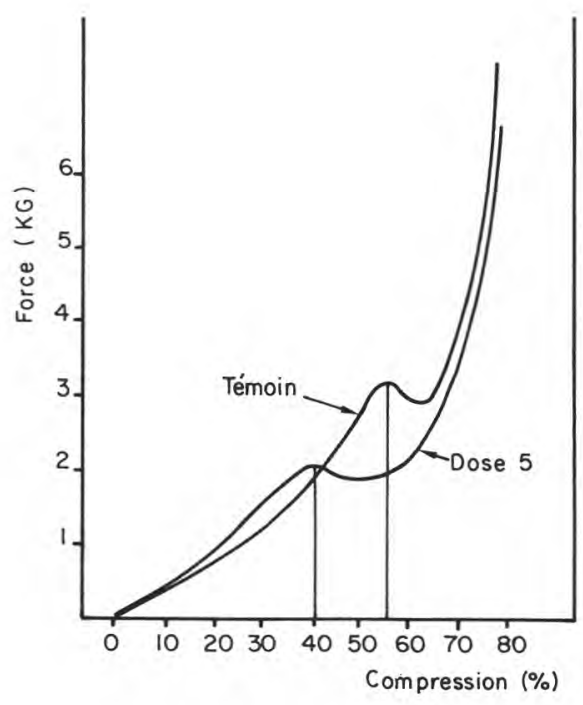

Fig. 5

Tests de compression: comparaison entre un fromage-témoin et un fromage ayant reçu une dose 5 de Rulactine. Les essais ont été effectués sur des fromages après 45 jours d'affinage.

Compression tests: comparison between control curd and with Rulactine (dose 5). Experiment was done after 45 days of ripening. 

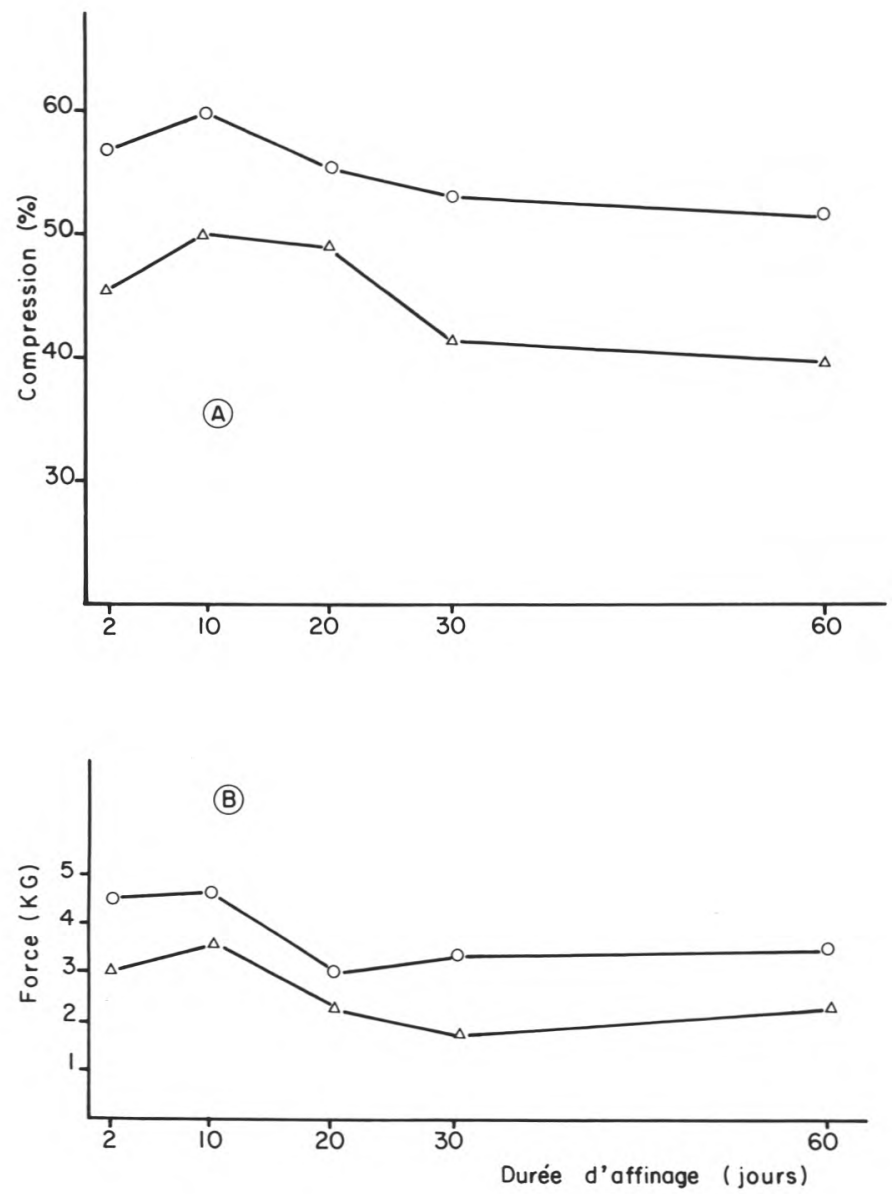

Fig. 6

Evolution des valeurs des taux de compression $(A)$ et de force $(B)$ à la rupture au cours de l'affinage.

Evolution of compression ratio $(A)$ and yield force $(B)$ during ripening.

\section{F. Amertume}

Le jury de dégustation n'a pas constaté d'apparition d'amertume dans les fromages-témoins et dans les fromages additionnés des doses 0,$5 ; 1$ et 2,5, ceci même après 45 jours d'affinage. Avec la dose 5, l'amertume devient clairement détectable après 30 jours d'affinage et pour les doses supérieures elle est présente dès 15 j d'affinage (fig. 7). 


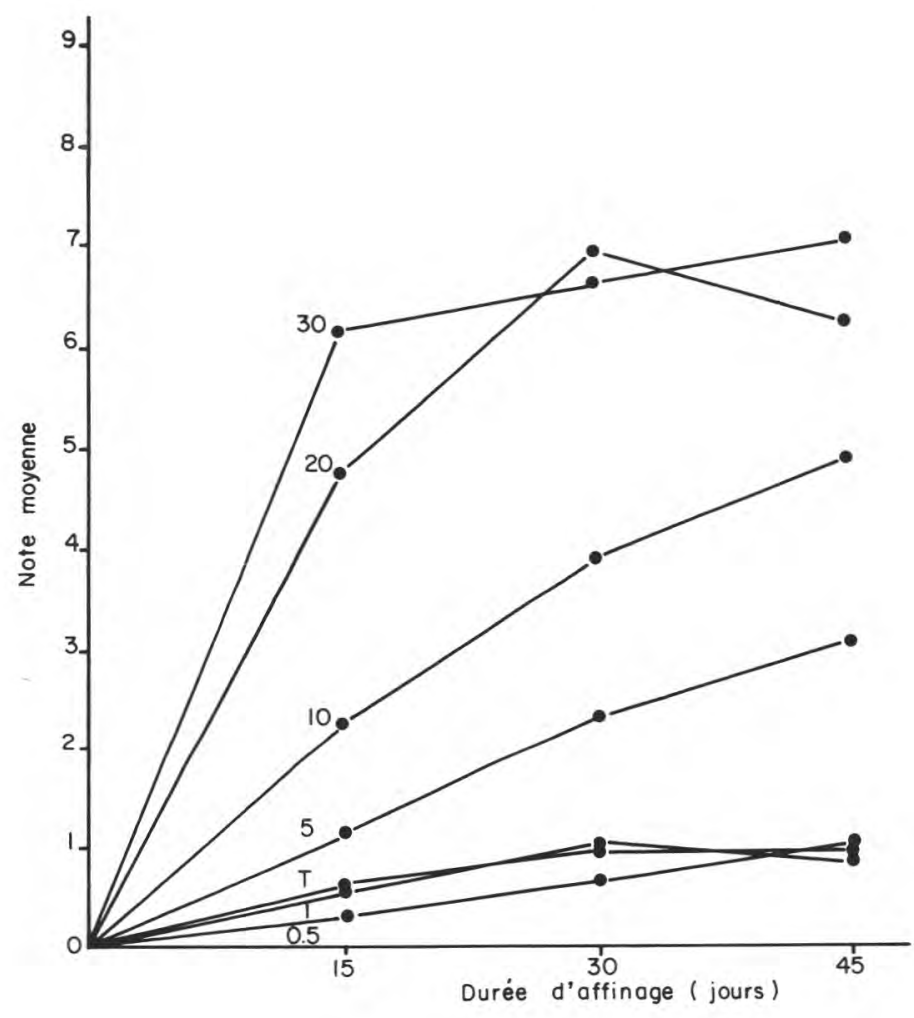

FIG. 7

Evolution de l'intensité de l'amertume au cours de l'affinage des fromages ayant reçu des doses croissantes de Rulactine $(T=$ fromage-témoin ; $1=$ dose 90 Unités/litre de lait ; $5=$ dose $450 \mathrm{U} / \mathrm{l} ; 10=$ dose $900 \mathrm{U} / \mathrm{l} ; 20=$ dose $1800 \mathrm{U} / \mathrm{l} ; 30=2700 \mathrm{U} / \mathrm{l}$ ).

Evolution of bitterness intensity during cheese ripening after addition of increased doses of Rulactine $(T=$ control curd $; l=$ dose 90 Unités $/ l$ of milk; $5=$ dose $450 \mathrm{U} / \mathrm{l} ; 10=$ dose $900 \mathrm{U} / \mathrm{l} ; 20=$ dose $1800 \mathrm{U} / \mathrm{l} ; 30=2700 \mathrm{U} / \mathrm{l})$.

\section{Discussion - Conclusions}

Le pourcentage de Rulactine retenu dans les caillés (13\%) est supérieur au pourcentage d'eau du lait $(\cong 6 \%)$ qui reste dans le fromage. Il existe donc une fixation de la préparation dans le caillé. Il faut toutefois noter que la Rulactine n'est pas une préparation d'enzyme pure. Le taux de rétention mesuré est donc celui d'un mélange et n'est pas nécessairement strictement identique à celui de l'enzyme pure.

La dose préconisée dans l'industrie pour l'utilisation de la Rulactine est de 90 Unités/litre de lait (dose 1). Dans nos conditions de fabrication et d'affinage cette dose ne provoque pas de différence avec les fromages-témoins que 
ce soit au niveau de la protéolyse dans les fromages, des caractères rhéologiques de la pâte ou de son goût. Avec la dose 2,5, une légère augmentation du degré de protéolyse est observée sans que cela se traduise par des modifications de goût ou de texture décelables. Avec la dose 5, une intensification nette de la protéolyse est obtenue et la texture de la pâte est modifiée et devient plus friable. La dose 2,5 (225 Unités/litre de lait) n'apporte pas de modification organoleptique détectable. Une dose double (dose 5 soit 450 Unités/litre) exerce un effet net sur la texture mais est accompagnée d'amertume et de perte de rendement azoté. Le créneau d'utilisation de cette enzyme correspondant à des doses provoquant une modification perceptible et positive de la texture sans apparition de défaut d'amertume et de perte de rendement est particulièrement étroit et l'intérêt de l'addition de Rulactine directement dans le lait de fromagerie apparaît donc très discutable.

Nos résultats soulignent que l'addition d'une protéase seule provoque un déséquilibre entre les différentes fractions azotées, générant de l'amertume même si les augmentations d'azote soluble restent assez limitées (dans notre cas, 6 à $7 \%$ de l'azote total du fromage). Ceci ne signifie pas qu'il faut exclure l'emploi de protéases. L'accélération de l'affinage nécessite en effet une protéolyse plus rapide, cette dernière provoquant les modifications de texture et participant à la formation des composés d'arôme. Toutefois, ceci souligne la nécessité d'employer des mélanges d'enzymes afin que le surplus de peptides amers produit par les protéases soit dégradé. Des mélanges protéases et peptidases ou protéases et broyats de cellules devraient permettre d'atteindre cet objectif. Cependant à notre connaissance il n'existe pas de peptidase industrielle de grade alimentaire disponible sur le marché. En fabrication de Cheddar, LAW et Wigmore (1983) ont utilisé un mélange protéase et extrait de Streptococcus lactis, ce dernier apportant, outre des aminopeptidases qui dégradent les peptides, d'autres enzymes pouvant participer à l'amélioration des qualités organoleptiques. L'addition de broyats bactériens conjointement aux protéases mérite donc d'être davantage étudiée.

Un autre inconvénient de l'emploi de protéases seules réside dans les pertes de rendement lorsqu'elles sont ajoutées directement au lait. Dans le cas du Cheddar, il est possible d'ajouter l'enzyme au caillé lors du salage ce qui évite les pertes d'azote dans le lactosérum (LAW et Wigmore, 1982 ; KosIKOWSKI et IWASAKI, 1975), mais ce procédé n'est pas utilisable pour d'autres fromages. L'encapsulation des enzymes dans de petites capsules phospholipidiques, les liposomes, est une solution qui a été proposée récemment (LAW et KING, 1985 ; PIARD et al., 1986). Il faut noter également que la majeure partie de la protéolyse due à l'addition de Rulactine dans le lait s'effectue au cours des 48 premières heures, l'augmentation au cours de l'affinage étant beaucoup plus lente. Les liposomes permettront peut-être d'obtenir une protéolyse plus progressive, en évitant une action trop importante au cours de la fabrication du caillé. 


\section{Remerciements}

Nous remercions vivement tous les collègues qui ont bien voulu participer aux différentes dégustations nécessitées par le jury ainsi que M. LE BARS pour la préparation de Rulactine marquée, $M^{\text {mes }}$ Martin et HoRTaut pour la dactylographie du manuscrit. Ce travail a bénéficié d'une aide financière dans le cadre d'une A.T.P.-I.N.R.A. (Utilisation des enzymes en fromagerie) et d'un contrat de programme I.N.R.A.-M.R.T. (Biotechnologie).

\section{Références bibliographiques}

Desmazeaud M.J., Hermier J., 1968a. Facteurs intervenant dans la production du système protéolytique chez Micrococcus caseolyticus. Ann. Biol. anim. Biochim. Biophys., 8, 419-429.

Desmazeaud M.J., Hermier J., 1968b. Isolement, purification et propriétés d'une protéase exocellulaire de Micrococcus caseolyticus. Ann. Biol. anim. Biochim. Biophys, , 8, 565-578.

Donnelly W.J., Barry J.F., Richardson T., $1980 .{ }^{14} \mathrm{C}$-methylated $\beta$-casein as a substrate for plasmin and its application to the study of milk protein transformations. Biochim. Biophys. Acta, 626, 117-126.

Gripon J.-C., Desmazeaud M.J., Le Bars D., Bergere J.L., 1975. Etude du rôle des microorganismes et des enzymes au cours de la maturation des fromages. II. Influence de la présure commerciale. Lait, 55, 502-516.

GRUDE P., 1978. Etude de la stimulation de la croissance de bactéries lactiques par les produits du métabolisme de Micrococcus caseolyticus dans le lait. Application à l'industrie fromagère. Thèse. Université de Dijon.

Koops J., Klomp H., Elgersma R.H.C., 1975. Rapid determination of nitrogen in milk and dairy products by colorimetric estimation of ammonia following an accelerated digestion procedure. Neth. Milk Dairy J., 29, 169-180.

KosıKowskı F.A., IWASAKI T., 1975. Changes in Cheddar cheese by commercial enzyme preparations. J. Dairy Sci., 58, 963-970.

LAW B.A., 1984. Flavour development in cheeses. In: Advances in the microbiology and biochemistry of cheese and fermented milk. Ed. Davies F.L., Law B.A., Elsevier Applied Science Publishers London, 187-208.

LAw B.A., KING J.S., 1985. Use of liposomes for proteinase addition to Cheddar cheese. J. Dairy Res., 52, 183-188.

Law B.A., Wigmore A., 1982. Accelerated cheese ripening with food grade proteinase. J. Dairy Res., 49, 137-146.

LAW B.A., WIgMORE A., 1983. Accelerated ripening of Cheddar cheese with a commercial proteinase and intracellular enzymes from starter streptococci. J. Dairy Res., 50, 519-525.

Maubors J.-L., MocQuot G., 1969. Action protéolytique d'un enzyme coagulant comparée à celle de la présure. Lait, 49, 497-506.

Piard J.C., El-Soda M., Alkhalaf W., Rousseau M., Desmazeaud M.J., Vassal L., Gripon J.C., 1986. Acceleration of cheese ripening with liposomes entrapped protease. Biotechnol. Lett., 8, 241-246.

SooD V.K., KosikowsKI F.V., 1979. Ripening changes and flavor development in microbial enzyme treated Cheddar cheese slurries. J. Food Sci., 44, 1690-1694.

Vassal L., Desmazeaud M.J., Gripon J.C., 1982. Modifications de fromages à pâte pressée par addition d'une métalloprotéase de Micrococcus caseolyticus. In: Utilisation des enzymes en technologie alimentaire. Ed. P. Dupuy, Paris, Technique et Documentation Lavoisier, 315-319. 